\title{
Endogenous Participation Risk in Speculative Markets
}

\author{
Edouard Challe* \\ CNRS-DRM (UMR 7088) and University of Paris-Dauphine \\ Place du Maréchal de Lattre de Tassigny, 75116 Paris, France
}

\begin{abstract}
This paper analyses the dynamic implications of an asset-pricing model with incomplete participation due to entry costs. It is shown that heterogeneity in entry costs can lead to the existence of multiple stochastic sunspot equilibria, whereby the number of agents in the market and asset prices fluctuate endogeneously over time in the absence of fundamental uncertainty. Such asset-price fluctuations occur despite the uniqueness of the deterministic equilibrium, and thus bear no link to the usual notion of steady-state indeterminacy. In addition to excess volatility, the equilibria exhibit predictable and conditionally heteroskedastic returns.
\end{abstract}

JEL classification: D84, G11

Keywords: incomplete participation; self-fulfilling expectations; asset price volatility
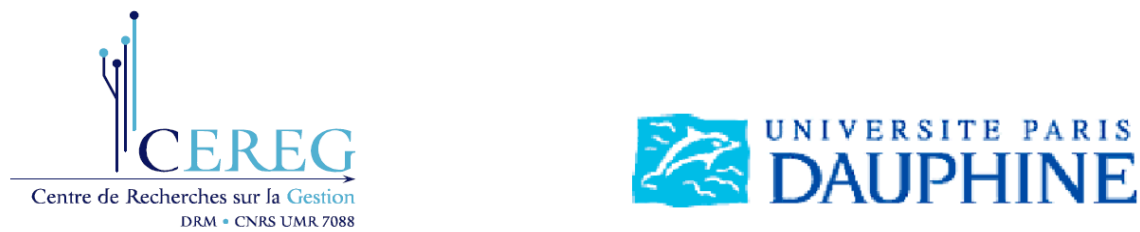

*Tel.: +33 (0)1 440545 65; Fax: +33 (0)1 440540 23; E-mail address: edouard.challe@dauphine.fr. 


\section{Introduction}

This paper analyses the impact of incomplete participation, due to the presence of entry costs, on the price dynamics of a speculative asset. It focuses on the possible destabilising nature of limited participation in asset markets, and shows that incomplete participation in the deterministic steady state may allow for the existence of stochastic equilibria where arbitrary, self-fulfilling, beliefs of investors cause fluctuations in both the number of agents in the market and asset prices.

From an empirical point of view, the fact that participation in some speculative markets (e.g., stock markets) is incomplete is fairly uncontroversial. This point was made by Mankiw and Zeldes (1991) to explain the cross-sectional patterns of returns on different assets (i.e., the equity premium puzzle). Moreover, there is considerable evidence that stock market participation varies over time, as shown by the dramatic increase in participation in Europe and the US during the 1990's (Guiso et al., 2003). At higher frequencies, a great number of empirical studies have shown that changes in asset-price volatility (as measured by daily squared price changes) are related to variations in trading volumes, with high trading volumes being associated with high volatility (e.g., Karpoff, 1987). Looking further into this correlation, Jones et al. (1994) established that it is almost entirely accounted for by changes in the number of transactions that take place in the market, rather than by changes in the size of individual transactions. To the extent that daily changes in the number of orders placed in a particular market reflect variations in the number of investors who are actually active in that market (rather than a pure recomposition of their orders), time-varying participation may be expected to substantially affect the dynamic behaviour of asset prices in the short run (besides its potential effect on the secular trend in prices and returns).

A natural explanation for incomplete market participation is that investors have to pay entry costs, which reflect fees, information-gathering costs, processing costs and the like, before they can convey buying or selling orders to the market. As a consequence, investors will weigh the expected utility gain from entering the market against the utility loss from paying this entry cost. If investors differ in terms of certain characteristics such as preferences, wealth, or the size of the entry costs to be paid, some agents will decide to participate while others may not, 
leading to situations of incomplete participation. If this explanation is correct, participation in speculative markets is driven by both entry costs and the risk/return trade-off investors face. Since returns and risk typically vary over time (e.g., Engle, 2004, Poterba and Summers, 1986 and 1988), we expect participation to be time-varying as well, and changing participation levels will in turn impinge on equilibrium prices, returns, and risk. As an example, Orosel (1998) shows that the price process resulting from incomplete participation typically magnifies the effect of dividend innovations by altering the flow of agents in and out of the market.

This paper shows that the feedback loop between asset prices and participation levels may have more dramatic implications. More precisely, heterogeneity in entry costs may lead to the existence of multiple, self-fulfilling stochastic equilibria whereby the ex ante expectation that prices will fluctuate generates a pattern of changing participation which, via its effect on equilibrium prices, validates this expectation ex post. To make this point clear, the framework introduced below assumes that the asset being traded bears no income risk, so that the only source of uncertainty lies in the volatility of investors' expectations.

The mechanism underlying such equilibria and their properties is reasonably intuitive. With mean-variance preferences (a decision criterion that approximates optimal choices in our model), high payoff volatility may depress the price of a risky asset to the extent that the resulting expected return is high enough to increase the expected utility from trading the asset (Pagano, 1989b). Now suppose that investors expect market participation to change over time. To attract a sufficiently large number of investors, so that participation is high, the (expected) risk-return trade-off must be more favorable relative to a state with lower participation. In our model, this property comes about when the high participation state has substantial payoff risk but relatively low asset prices and high expected returns, the prospect of which sufficiently increases the attractiveness of the asset to compensate for the greater risk. Conversely, states with relatively low investor participation have low payoff risk but relatively high prices and low expected returns, which latter deter investors with high entry costs from entering the market. As subsequently will be shown, for this mechanism to work we require the stochastic sunspot process behind the market participation dynamics (and thus equilibrium asset prices) to be sufficiently heteroskedastic to bring about substantial differences in the conditional variance of asset payoffs across states. When this is the case, expectations of changes in the participation 
level become self-fulfilling and create a payoff risk, which we label endogenous participation risk, which occurs independently of that associated with the asset's income. This mechanism is one possible cause of excess volatility in asset prices, since no fundamental uncertainty is needed for prices to fluctuate randomly. It will be shown that these equilibria also exhibit some well-known properties of empirical asset returns, such as predictability and conditional heteroskedasticity (i.e. time-varying risk).

A number of authors have investigated the implications of incomplete participation for the multiplicity of equilibria in asset markets. In a pioneering contribution, Pagano (1989a) analysed the negative feedback between market volatility and the level of market participation. High participation lowers the impact of idiosyncratic demand shocks on asset prices, thereby reducing asset price volatility and making participation more attractive. Multiple steady states can then appear as the mere expectation of high participation levels attracts more investors, whereas expectation of low participation deters investors from participating. In a related article, Allen and Gale (1994) considered the impact of aggregate liquidity shortages on the existence and properties of asset market equilibria. Both papers focus on the volatility and welfare implications of (possibly multiple) participation levels, i.e., the steady states of the corresponding models. In contrast, we here analyse the possibility of self-fulfilling stochastic equilibria (sunspot equilibria), where the steady state is unique and the only source of uncertainty is extraneous.

The possibility of sunspot equilibria in asset markets has long been recognised in dynamic macroeconomic models plagued with market imperfections. For example, Aiyagari (1988) and Azariadis and Chakraborty (1998) argue that sunspot equilibria offer a natural theory of excess volatility in asset prices, whereas Challe (2004) shows that they typically imply that asset returns are predictable. There are two dimensions in which the model developed below differs from this previous work. From a theoretical point of view, our model relates self-fulfilling asset price volatility to the flows of agents in and out of speculative markets. The model is thus rooted in the market microstructure approach to market participation (e.g., Pagano, 1989a and 1989b), as opposed to the dynamic general equilibrium framework favoured by macroeconomists. ${ }^{1}$ Methodologically, our approach differs from that in previous contributions (including

\footnotetext{
${ }^{1}$ Of course, others factors than entry cost may produce time-varying participation and asset prices. For example, it has been recently argued that binding and time-varying borrowing constraints may restrict the
} 
Aiyagari, 1988, and Challe, 2004), which generate asset-price sunspots within dynamic models displaying indeterminacy, that is, a multiplicity of dynamic equilibrium trajectories converging towards the steady state. As is well-known from the literature on capital accumulation and growth (e.g., Benhabib and Farmer, 1994 and 1998), indeterminacy is a sufficient condition for sunspot fluctuations to arise in dynamic models as it provides the economy with the multiple (deterministic) equilibria on which agents may randomly coordinate their actions at any point in time. In contrast, in our approach the kind of self-fulfilling expectations that may occur arise independently from (and, in fact, despite the lack of) steady-state indeterminacy.

Section 2 introduces the model and derives its deterministic equilibrium. Section 3 proves the existence of equilibria with endogenous participation risk and analyses their volatility properties. Section 4 provides a worked example of stochastic equilibrium, and Section 5 concludes.

\section{The model}

\subsection{Assets and market structure}

The analytical framework consists of an overlapping generations model in which investors who live for two periods maximise the expected utility of final consumption (i.e., they do not consume in the first period of their life). Investors entering the market at date $t$ receive an endowment $e>0$ and may trade two assets. One is a safe asset in perfectly elastic supply that can be used to borrow or lend without limit at a gross interest rate of $R>1$. The other asset (referred to as 'the asset' in the remainder of the paper) is in fixed supply (normalised to 1) and yields a constant dividend $d$ per share in every period. The price of the asset at date $t$ is denoted $p_{t}$.

There is a continuum of investors of mass 1 indexed by $i$ and uniformly distributed along the interval $[0,1]$. Before entering the market for risky assets and issuing buying or selling orders there, investor $i$ must pay a fixed cost $\epsilon(i) \geq 0$ (no entry cost is required in order to trade the

househoulds' ability to trade assets optimally and affect equilibrium prices (e.g., Azariadis and Kaas, 2007, and Constantinides et al., 2002). Our paper differs from this approach in terms of both the source of participation changes, and the way participation affects prices. 
safe asset). The budget constraint of investor $i$ at date $t$ is thus

$$
p_{t} X_{t}^{i}+M_{t}^{i}+z \epsilon(i) \leq e
$$

where $X_{t}^{i}$ is investor $i$ 's demand for risky assets, $M_{t}^{i}$ is the investor's demand for safe assets, and $z$ is a dummy variable taking the value 1 if the investor decides to enter the market for risky assets, and 0 otherwise. From the budget constraints of investor $i$ at dates $t$ and $t+1$, the terminal consumption of investor $i$ is given by:

$$
\begin{aligned}
C_{t+1}^{i} & =M_{t}^{i} R+X_{t}^{i}\left(p_{t+1}+d\right) \\
& =R(e-z \epsilon(i))+X_{t}^{i}\left(p_{t+1}+d-R p_{t}\right)
\end{aligned}
$$

The equilibria considered in this paper are in general stochastic and involve fluctuations in the asset price. The resulting payoff risk is measured by the conditional variance of asset prices, $\sigma_{t}^{2} \equiv \operatorname{var}_{t}\left(p_{t+1}\right)$.

\subsection{Preferences and entry decisions}

Investors are endowed with constant absolute risk aversion (CARA) utility functions. Let $\gamma>0$ denote the common risk-aversion coefficient of investors, and $\bar{C}^{i}$ the unconditional mean of $C_{t}^{i}$ (i.e., the mean consumption of investors with entry cost $\epsilon(i))$. In the remainder of the paper, we shall consider fluctuations in asset prices (and thus in asset payoffs and individual consumption levels) which take place over a small, continuous support. Therefore, we can follow Levy and Markovitz (1979) and consider the quadratic approximation to the utility function around $\bar{C}^{i}$ to derive the following local mean-variance criterion: ${ }^{2}$

$$
\max E_{t}\left(-\exp \left(-\gamma C_{t+1}^{i}\right)\right) \simeq a^{i}+b^{i}\left(E_{t}\left(C_{t+1}^{i}\right)-\frac{\gamma}{2} \operatorname{Var}_{t}\left(C_{t+1}^{i}\right)\right)
$$

\footnotetext{
${ }^{2}$ The mean-variance criterion has sometimes been criticised on the grounds that it may lead agents to invest into stochastically dominated assets (e.g., Hanoch and Levy, 1969). In our model, prices are trend-reverting and the expected return to risky assets always exceeds the safe rate, so this would happen if the buying price of the asset were so low that the risky asset were bound to do better than the safe asset ex post (due to the potential capital gains involved). However, this cannot occur if some uncertainty with arbitrarily small variance but unbounded support is added to dividends or the asset supply, as is generally assumed in overlapping generations models with mean-variance investors (through the use of normally distributed payoff risk, e.g., Hellwig, 1982, Pagano, 1989a, and Spiegel, 1998).
} 
where $a^{i} \equiv-(1+\gamma) \exp \left(-\gamma \bar{C}^{i}\right)$ and $b^{i} \equiv \gamma \exp \left(-\gamma \bar{C}^{i}\right)$. Note that $\bar{C}^{i}$ (and thus $a^{i}$ and $\left.b^{i}\right)$ will typically vary across agents, due to heterogeneity in entry costs and entry decisions. However, the utility function we have chosen ensures that optimal portfolios depend only on $\gamma$, and are thus independent of $\bar{C}^{i}$.

When deciding whether to enter the market, investors solve a two-stage decision problem (e.g., Pagano, 1989a). First, they compute their (notional) optimal asset demand conditional on participation, and the associated level of expected utility, $U P_{t}(i)$; and second, they compare this level of utility to that associated with not participating, $U N_{t}(i)$.

First stage. Assume that $\sigma_{t}^{2}>0$ (the case with $\sigma_{t}^{2}=0$ is the deterministic equilibrium analysed in Sec. 2.3 below). If investor $i$ decides to enter the market, then from Eqs. (1)-(2) optimal asset demand is

$$
\hat{X}_{t}^{i}=\frac{E_{t}\left(p_{t+1}\right)+d-R p_{t}}{\gamma \sigma_{t}^{2}} .
$$

Second stage. Investor $i$ participates in the asset market if the expected utility from participation (i.e., setting $z=1$ and $\left.X_{t}^{i}=\hat{X}_{t}^{i}\right)$ is greater than that from not participating $\left(z=X_{t}^{i}=0\right)$. From Eqs. (1) and (2), the latter is simply $U N_{t}(i)=a^{i}+b^{i} e R$. Using Eqs. (1)-(3), the expected utility from participation is

$$
U P_{t}(i)=a^{i}+b^{i}\left(R(e-\epsilon(i))+\frac{\left(E_{t}\left(p_{t+1}\right)+d-R p_{t}\right)^{2}}{2 \gamma \sigma_{t}^{2}}\right) .
$$

Investor $i$ then participates if, given the current asset price and the conditional distribution of the asset payoff, $U P_{t}(i) \geq U N_{t}(i)$, or:

$$
\frac{\left(E_{t}\left(p_{t+1}\right)+d-R p_{t}\right)^{2}}{2 R \gamma \sigma_{t}^{2}} \geq \epsilon(i) .
$$

Finally, let investors be ordered along $[0,1]$ such that $\epsilon($.$) is non-decreasing. Given the entry$ condition (5), if investor $i^{*}$ wishes to enter the market for a given conditional payoff distribution, $E_{t}\left(p_{t+1}+d\right), \sigma_{t}^{2}$, then all investors $i<i^{*}$ will also wish to do so. Moreover, it is assumed that $\epsilon($.$) is continuous over [0,1]$, that there exist $\tilde{\imath} \in(0,1)$ such that $\epsilon(i)=0$ for $i \leq \tilde{\imath}$ (i.e., there is a positive mass of investors with zero entry costs), and that $\epsilon(i)$ is positive and strictly increasing in $i$ for $i>\tilde{\imath}^{3}$

\footnotetext{
${ }^{3}$ Assuming that there is a positive mass of investors with zero entry costs is essential for the deterministic
} 


\subsection{Deterministic equilibrium}

It is useful to derive, as a benchmark case, the deterministic equilibrium of this economy. In this equilibrium, $\operatorname{Var}_{t}\left(C_{t+1}^{i}\right)=\sigma_{t}^{2}=0$ and preferences become linear in final consumption. Prices are then given by a risk-neutral, no-arbitrage condition according to which agents facing zero entry costs trade the asset until its gross return is equal to $R$. Using (1)-(2), this condition is:

$$
p_{t+1}+d-R p_{t}=0
$$

The unique non-explosive price path of the deterministic economy is then:

$$
\bar{p}=d /(R-1), t=0,1, \ldots,
$$

which we may term the fundamental value of the asset, i.e., the price that would prevail if no extraneous uncertainty affected investors' beliefs. Note that, along the deterministic equilibrium, investors facing positive entry costs never enter the market, because their return from trading the asset, net of entry cost, is strictly less than $R$.

\section{Equilibria with endogenous participation risk}

In what follows we first venture, and then prove the existence of, equilibria where market participation and asset prices fluctuate according to arbitrary (but ultimately rational) expectations of investors. We thus proceed by construction. First, we assume that investors may rationally expect market participation at date $t, i_{t}$, to fluctuate over time. Second, we derive the conditional distribution, $\left(E_{t}\left(p_{t+1}\right), \sigma_{t}^{2}\right)$, that prices must obey for the market to clear given current participation, $i_{t}$. And third, we provide a simple process for participation dynamics that satisfies equilibrium conditions.

\subsection{Market clearing and equilibrium prices}

Market clearing requires that total market demand for the asset be equal to 1 when the (possibly time-varying) level of participation is $i_{t}$. Using Eq. (3), individual asset demands can be equilibrium computed in Sec. 2.3. to be well defined. Dropping this assumption (while maintaining the continuity of the $\epsilon$ (.) function) leads to an economy where only stochastic equilibria may exist. 
aggregated across participating investors to obtain, for all dates:

$$
\int_{0}^{i_{t}} \hat{X}_{t}^{i} d i=i_{t}\left(\frac{E_{t}\left(p_{t+1}\right)+d-R p_{t}}{\gamma \sigma_{t}^{2}}\right)=1 .
$$

Note first that Eq. (8) can be used to derive the expected net payoff accruing to investors when participation is $i_{t}$ and the conditional variance of asset prices is $\sigma_{t}^{2}$ :

$$
E_{t}\left(p_{t+1}\right)+d-R p_{t}=\gamma \sigma_{t}^{2} / i_{t}
$$

Eq. (9) summarises the risk-return tradeoff faced by investors in equilibrium. When the risk associated with holding the asset is high (low), the asset commands a relatively high (low) expected net payoff. The impact of market participation, $i_{t}$, on this tradeoff reflects the usual effect of the number of investors on risk-sharing in incomplete participation models (e.g., Pagano, 1989b). More specifically, for a given variance $\sigma_{t}^{2}$, higher participation leads to a smaller proportion of risky assets in any participating investor's portfolio (given the fixed supply of risky assets), and thus lower values of both the portfolio risk and the required consumption premium.

Using (9) allows us to substitute prices out of the entry condition (5). We find that any stochastic equilibrium must satisfy the following inequalities, for all $t \geq 0$ :

$$
\begin{aligned}
\gamma \sigma_{t}^{2} / 2 R i_{t}^{2} & \geq \epsilon(i) \text { for } i \leq i_{t} \\
& <\epsilon(i) \text { for } i>i_{t}
\end{aligned}
$$

The left-hand side of (10) is the utility gain that an investor expects from joining the asset market rather than staying out of it when current participation is $i_{t}$. For the decisions of investors as a whole to generate a participation level of $i_{t}$, it must be the case that only investors facing entry cost less than (or equal to) $\epsilon\left(i_{t}\right)$ find it worthwhile to join, while those facing $\epsilon(i)>\epsilon\left(i_{t}\right)$ prefer to stay out (the right-hand side of (10)). Given the assumed continuity of $\epsilon($.$) , it follows$ that (10) must hold as an equality for the last entrant, or marginal investor at date $t$, who is precisely indifferent between entering and staying out of the market when participation is $i_{t}$. This implies that along any stochastic equilibrium the conditional variance of asset prices and the level of participation are related as follows:

$$
\sigma_{t}^{2}=2(R / \gamma) i_{t}^{2} \epsilon\left(i_{t}\right)
$$


Note that when $\sigma_{t}^{2}=0$ we have again the deterministic equilibrium of Sec. 2.3, where participating agents are in positive mass (i.e. $i_{t}=\tilde{\imath}>0$ ), but all face zero entry costs (i.e., $\epsilon(i)=\epsilon(\tilde{\imath})=0)$. Solving (9) for $p_{t}$ and using (11), we find that current asset prices depend on current participation and future prices as follows:

$$
p_{t}=\left(E_{t}\left(p_{t+1}\right)+d\right) / R-2 i_{t} \epsilon\left(i_{t}\right)
$$

Finally, iterating (12) forwards under the no-bubble condition $\lim _{n \rightarrow \infty} E_{t}\left(p_{t+n}\right) / R^{n}=0$, the equilibrium price of the asset at date $t$ can be written as a function of current and future levels of participation:

$$
p_{t}=\frac{d}{R-1}-2 E_{t}\left(\sum_{j=0}^{\infty} R^{-j} i_{t+j} \epsilon\left(i_{t+j}\right)\right)
$$

In the deterministic equilibrium, $i_{t}=\tilde{\imath}$ and $\epsilon\left(i_{t}\right)=0$ for all $t \geq 0$, so that (13) collapses to (7). Fluctuations in participation levels, if possible, must thus involve values of $i_{t}$ higher than $\tilde{\imath}$ for some $t$, which in turn would cause fluctuations in asset prices according to Eq. (13). ${ }^{4}$

\subsection{Existence of stochastic equilibria}

The question of whether stochastic equilibria exist amounts to asking whether there exists nondegenerate stochastic processes for market participation, $\left\{i_{t}\right\}_{t=0}^{\infty}$, asset prices, $\left\{p_{t}\right\}_{t=0}^{\infty}$, and conditional variance, $\left\{\sigma_{t}^{2}\right\}_{t=0}^{\infty}$, where $\sigma_{t}^{2}=\operatorname{var}_{t}\left(p_{t+1}\right)$, that satisfy the market-clearing condition $(8)$, the payoff-variance condition (11) and the boundary condition $i_{t} \in(0,1]$, for all $t \geq 0$. The following proposition establishes that this is the case.

Proposition 1 (Existence). There exist equilibria where participation and asset prices change over time (i.e., where $\sigma_{t}^{2}>0$ ) provided that $\gamma \epsilon(1)>2 R$. Along such equilibria, asset prices always stay below their fundamental value, $\bar{p}=d /(R-1)$.

Proof. The proof is by construction. Define the function $I_{t}=f\left(i_{t}\right) \equiv 2 i_{t} \epsilon\left(i_{t}\right)$, and notice that $f($.$) is continuous and that f\left(i_{t}\right)=0$ for $i_{t} \leq \tilde{\imath}$ while $f\left(i_{t}\right)>0, f^{\prime}\left(i_{t}\right)>0$ for $i_{t}>\tilde{\imath}$. Assume

\footnotetext{
${ }^{4}$ Since $i_{t} \in[0,1], E_{t}\left(\sum_{j=0}^{\infty} R^{-j} i_{t+j} \epsilon\left(i_{t+j}\right)\right)$ is bounded above, so $d$ can always be set to ensure that equilibrium prices are non-negative at all times.
} 
that $I_{t} \in(0,2 \epsilon(1)]$ (that is, $i_{t}>\tilde{\imath}$ for all $t \geq 0$ ), and that $I_{t}$ obeys the following process:

$$
I_{t}=\bar{I}+F\left(I_{t-1}\right) \eta_{t}
$$

where $\bar{I}=2 \bar{\imath} \epsilon(\bar{\imath})$ is the unconditional mean of $I_{t}, F($.$) is a sensitivity function (to be specified),$ and $\left\{\eta_{t}\right\}_{t=0}^{\infty}$ is a white noise process with symmetric distribution, bounded support $[-\hat{\eta}, \hat{\eta}]$, $\hat{\eta}>0$, and such that $E_{t}\left(\eta_{t+1}\right)=0$ and $\operatorname{var}_{t}\left(\eta_{t+1}\right)=1$. Substituting (14) into (12)-(13) and rearranging, we may rewrite the current price of the asset as follows:

$$
p_{t}=(d-\bar{I}) /(R-1)-I_{t}
$$

From (14)-(15), the conditional variance of asset prices is $\sigma_{t}^{2}=\operatorname{var}_{t}\left(I_{t+1}\right)=F\left(I_{t}\right)^{2}$. Now, $\sigma_{t}^{2}$ must also satisfy Eq. (11) for the implied price process to be an equilibrium. Rewriting $\sigma_{t}^{2}$ in (11) as $R I_{t} f^{-1}\left(I_{t}\right) / \gamma$ and equating it to $F\left(I_{t}\right)^{2}$, we find that the sensitivity function must be:

$$
F\left(I_{t}\right)=\sqrt{R I_{t} f^{-1}\left(I_{t}\right) / \gamma}
$$

where $F\left(I_{t}\right)$ is strictly increasing in $I_{t}$.

Lastly, the feasibility of stochastic equilibria requires that $I_{t}$ stay within the interval $(0,2 \epsilon(1)]$ for all $t$. From (14), $I_{t}$ is bounded above if

$$
I=\bar{I}+F(I) \hat{\eta}
$$

has a unique, positive and finite solution $I^{*}$ (the upper bound $I^{*}$ may be seen as the limit of $I_{t}$ as the innovation $\eta_{t}$ takes its maximium value $\hat{\eta}$ infinitely many times). Using (16), (17) can be rewritten as $\mu(I)=\nu(I)$, where $\mu(I) \equiv 1-\bar{I} / I$ and $\nu(I) \equiv \hat{\eta} \sqrt{R f^{-1}(I) / \gamma I}$. Both $\mu($.$) and$ $\nu($.$) are continuous over [\bar{I}, 2 \epsilon(1)]$, while $\mu(\bar{I})=0$ and $\nu(\bar{I})>0$. Thus, (17) has at least one solution $I^{*} \in(\bar{I}, 2 \epsilon(1))$ if $\nu(2 \epsilon(1))<\mu(2 \epsilon(1))$, that is, if

$$
\gamma>\hat{\gamma}=2 \epsilon(1) R \hat{\eta}^{2} /(2 \epsilon(1)-\bar{I})^{2}
$$

Moreover, this solution is unique since $\mu$ (.) is strictly increasing and $\nu($.$) is strictly decreasing.$ To establish this latter point, note that $\nu^{\prime}(I)<0$ is equivalent to $I \partial f^{-1}(I) / \partial I<f^{-1}(I)$. As $I=f(i)=2 i \epsilon(i)$ (and thus $\left.\partial f^{-1}(I) / \partial I=1 / f^{\prime}(i)=1 /\left(2 \epsilon(i)+2 i \epsilon^{\prime}(i)\right)\right), \nu^{\prime}(I)<0$ is equivalent to $\epsilon^{\prime}(i)>0$ (which is true for $i>\tilde{\imath}$ by assumption). 
Given the maximum possible value taken by the sensitivity function, $F\left(I^{*}\right)$, and the fact that $\eta$ has lower bound $-\hat{\eta}$, it follows that $I_{t} \geq I_{*}=\bar{I}-F\left(I^{*}\right) \hat{\eta}$ for all $t$ (i.e., $I_{t}$ is bounded below by $\left.I_{*}\right) . I^{*}$ solves $(17)$, so the condition $I_{*}>0$ is equivalent to $2 \bar{I}>I^{*}$; and since $I^{*}<2 \epsilon(1)$, a sufficient condition for $2 \bar{I}>I^{*}$ is that $\bar{I} \geq \epsilon(1)$. We look for the smallest possible value of $\hat{\gamma}$ in (18) guaranteeing the feasibility of stochastic equilibria. Amongst the class of symmetric distributions with support $[-\hat{\eta}, \hat{\eta}]$ and satisfying $E_{t}\left(\eta_{t+1}\right)=0, \operatorname{var}_{t}\left(\eta_{t+1}\right)=1$, the one with the lowest $\hat{\eta}$ is $\eta_{t}=1$ w.p. $1 / 2, \eta_{t}=-1$ w.p. $1 / 2($ so that $\hat{\eta}=1)$. Then, setting $\hat{\eta}=1$ and $\bar{I}=\epsilon(1)$ in (18) gives $\hat{\gamma}=2 R / \epsilon(1)$. Finally, from (11) and the fact that $\sigma_{t}^{2}>0$, we have that $i_{t} \epsilon\left(i_{t}\right)>0$; then, Eq. (13) implies that prices are always less than $d /(R-1)$

The intuition for the sufficient condition stated in proposition 1 can be understood from Eq. (11), which relates the conditional variance of prices to the level of participation. In short, Eq. (11) states that low risk aversion coefficients, $\gamma$, or high values of the riskless rate, $R$, would generate stochastic equilibria with a relatively high variability of next period's prices, $p_{t+1}$, given current participation, $i_{t}$. Now, from Eq. (13), the conditional variance of prices is positively related to that of next period's participation. Since the variability of future participation must be small for participation to stay within the required interval, $\gamma$ must be sufficiently high, and/or $R$ sufficiently low, for stochastic equilibria to exist. Finally, that prices fluctuate below their fundamental value is an immediate consequence of the positive risk premium required by investors entering a market where participation risk affects prices.

It is important to notice that the kind of self-fulfilling fluctuations occurring in our framework differ from the indeterminacy and sunspot types traditionally used when analysing the stability properties of infinite-horizon models. In those models, stochastic equilibria are typically constructed by randomising over a local infinity of deterministic trajectories converging towards the steady state, along the lines described in Woodford (1986). The generality of this latter approach has led to its widespread use in a variety of dynamic settings including growth, monetary theory, and business cycles - see Benhabib and Farmer (1999) for a general overview, as well as Aiyagari (1988), Azariadis and Chakraborty (1998), and Challe (2004) for applications to asset pricing issues. The mechanism underlying asset-price fluctuations in our model is qualitatively different from this approach, since our deterministic equilibrium is unique (see Sec. 2.3). In our model, the 
possibility of self-fulfilling asset-price fluctuations is related to the dynamic interactions between risk and returns in equilibrium, rather than jumps across deterministic paths that may occur in dynamic models with indeterminate steady states. These interactions are described in more detail in the next Section.

\subsection{Properties of stochastic equilibria}

Although Eqs. (14)-(16) may potentially generate a large set equilibria with time-varying participation levels (depending on $\bar{I}$ and the distribution of $\eta$ ), all such equilibria share some robust properties that allow for an intuitive interpretation of the mechanism at work. These properties are summarised in the following proposition.

Proposition 2 (Prices, returns and risk). Along the stochastic equilibria defined by Eqs. (14)-(16), the higher is current participation, $i_{t}$, the lower is the asset price, $p_{t}$, and the higher are both the expected net payoff, $E_{t}\left(p_{t+1}\right)+d-R p_{t}$, and the payoff variance, $\sigma_{t}^{2}$.

Proof. That high (low) participation is associated with high (low) payoff risk follows from (11), while the negative relation between asset prices and participation can be seen from Eq. (15) and the fact that $I_{t}$ is a continous, increasing function of $i_{t}$ when $i_{t}>\tilde{\imath}$. Substituting (11) into (9) shows that the expected net payoff must be high (low) when participation is itself high (low)

In other words, stochastic equilibria with changing participation are characterised by conditional heteroskedasticity, i.e., time-variation in the conditional variance of the asset payoff. Since varying participation levels are accompanied by fluctuations in asset prices in the absence of income risk, prices also feature excess volatility. Finally, the fact that investors expect high returns when participation is high and low returns when participation is low implies that returns are predictable. These three properties are closely connected. It is precisely because the risk associated with holding the asset is relatively high when participation is high that the price must then be low, in spite of the larger number of market participants. At this point we may wonder why a state where the asset is high-risk attracts more participants than a state where it is low-risk. Would not the lower risk associated with the asset when participation is low also attract investors who are willing to buy the asset when participation is higher? The reason for 
this apparent paradox is that, for a certain level of risk, agents facing high entry costs require a higher return to participate than agents facing comparatively low costs. When participation is high, high risk causes the current price to be low, implying that expected returns are high, and high enough to make it worthwhile for a relatively large number of investors to participate despite the high associated risk. Conversely, when participation is low then the price of the asset is high, making expected returns low enough to deter many agents from participating despite the low associated risk.

Such times-series properties of asset prices have long been documented in empirical work. The excess volatility of asset prices (or, to be more precise, their high volatility relative to that of dividends), as well as the close connection between volatility and the predictability of asset returns, are now well-established and understood following the work of Shiller (1981) and Campbell and Shiller (1989) (see also Challe, 2004, and Poterba and Summers, 1988). On the other hand, fluctuations in prices and returns characterised by time-varying, persistent volatilities are at the root of the empirical success of dynamic volatility models in finance (see Poterba and Summers, 1986, for an early contribution, and Engle, 2004, for a recent overview of this approach).

In our model, these fluctuations are related to changes over time in the number of investors who actively participate in the market for risky assets, higher participation being associated with higher price volatility. Note that the empirical literature on asset-price formation has pointed out such a correlation. First, daily absolute or squared price changes -two typical measures of asset-price volatility in the empirical microstructure literature- have long been recognised to be positively related to transaction volume (this literature is surveyed in Karpoff, 1987). And second, virtually all of the time-variation in transaction volumes is explained by changes in the number of transactions that take place in the market, rather than by changes in the size of orders placed in the market by investors, as predicted by asset-pricing models with constant participation (see Jones et al., 1994). Since the number of such orders can arguably be interpreted as a proxy for the number of investors who are actually active in the market, the relation between volatility and participation implied by the model seems to be broadly consistent with the evidence. ${ }^{5}$

\footnotetext{
${ }^{5}$ Note that lower participation in one market does not necessarily mean lower participation in risky asset
} 


\section{An example}

As a numerical illustration of propositions 1 and 2 , take $\gamma=8, e=d=10, R=1.05$, and the following continuous cost function (plotted in the first panel of Figure 1):

$$
\epsilon(i)=\left\{\begin{array}{l}
0 \text { for } i \in[0,0.1] \\
2 i-0.2 \text { for } i \in(0.1,0.2] \\
i \text { for } i \in(0.2,1]
\end{array}\right.
$$

We consider fluctuations in $i_{t}$ that obey the stochastic process characterised by Eqs. (14)-(16), and compare them to the deterministic equilibrium. From Eq. (7) and the assumed cost function, the level of asset prices and participation along the deterministic equilibrium are $\bar{p}=200$ and $i_{t}=0.1$ for all $t$. To construct the stochastic equilibrium, focus on the case where $i_{t} \in(0.2,1]$ (so that $I_{t} \equiv 2 i_{t} \epsilon\left(i_{t}\right)=2 i_{t}^{2} \in(0.08,2]$, and take $\bar{\imath}=\sqrt{0.5}$ (so that $\bar{I}=1=\epsilon(1)$ ). From Eqs. (11) and (15), asset prices fluctuate around the value $(d-I R) /(R-1)=179<200$, while their conditional variance oscillates around $2(R / \gamma) \bar{\imath}^{3}=0.0928>0$. From Eqs. (14) and (16), as well as our chosen cost function and value of $\bar{I}$, the dynamics of $I_{t}$ can be written as:

$$
I_{t}=1+\left(\sqrt{R /\left(2^{1 / 2} \gamma\right)} \times I_{t-1}^{3 / 4}\right) \eta_{t}
$$

where the innovation process, $\left\{\eta_{t}\right\}$, is here assumed to be uniformly distributed over $[-\sqrt{3}, \sqrt{3}]$ (that $\hat{\eta}=\sqrt{3}$ implies that $E_{t}\left(\eta_{t+1}\right)=0$ and $\operatorname{var}_{t}\left(\eta_{t+1}\right)=1$, as our normalisation requires). In the present example, the conditions on the boundedness of $I_{t}$ are more stringent that in the general case studied in the proof proposition 1 , for two reasons. First, $i_{t}$ must stay within the interval $(0.2,1]$ (i.e., where $\epsilon(i)=i$ ); the relevant lower bound for $I_{t}$ is thus 0.08 , rather than 0 . And second, our sufficient condition $\gamma \epsilon(1)>2 R$ was derived using the lowest possible value of $\hat{\eta}(=1)$, given the assumed first and second moments of $\eta_{t}$, while the example has $\hat{\eta}=\sqrt{3}>1$. However, our chosen parameters do guarantee that $I_{t}$ stays within the interval required for the feasibility of the equilibrium. From Eq. (17), the upper bound $I^{*}$ is the unique solution to:

$$
I=1+\left(\sqrt{R /\left(2^{1 / 2} \gamma\right)} \times I^{3 / 4}\right) \hat{\eta}
$$

markets generally. In reality, portfolio rebalancing by investors may lead lower participation in one market to increase participation in others. Of course, our model precludes this possibility by assuming a single risky asset. 
which gives $I^{*}=1.8303(<2$, as required $)$. The lower bound is then $I_{*}=1-\sqrt{3 R / \gamma 2^{1 / 2}} \times I^{*} 3 / 4=$ $2-I^{*}=0.1697$ ( $>0.08$, as required). Thus, setting $I_{0}=\bar{I}=1$, an infinite sequence of $I_{t}$ starting from $I_{1}$ can be generated using (19), that will satisfy all equilibrium conditions. The implied values of $i_{t}, \sigma_{t}^{2}$ and $p_{t}$ naturally follow from Eqs. (11)-(13). The second panel of Figure 1 plots the negative relation between prices (solid line, left scale) and conditional variances (dotted line, right scale) for a particular draw of sunspot shocks over 200 periods.

\section{FIGURE 1 HERE}

\section{Concluding remarks}

This paper analyses the link between the presence of entry costs in the market for a speculative asset and the existence of self-fulfilling, stochastic equilibria with time-varying participation levels and asset prices. In these equilibria, the ex ante expectation that prices will fluctuate over time generates a time-pattern of participation in the market that validates this original expectation ex post, even though the asset bears no income risk. This model has been shown to be consistent with some well-known stylised stock-market facts, such as the excess volatility of asset prices as well as the predictability and conditional heteroskedasticity of asset returns.

The results have been derived under the restrictive assumption of homogenous, constant absolute risk aversion preferences. Most results carry over in a more general set-up that retains the CARA assumption whilst dropping that of homogenous risk-aversion, provided that some minimal continuity assumptions about agents' types are made (see the Appendix for a sketch of this generalisation). We expect similar stochastic equilibria to exist under more general utility functions, although the specific conditions under which this conjecture is true remain to be established. 


\section{Acknowledgement}

I am grateful for comments received from three anonymous referees, as well as from seminar participants at the Universities of Cambridge, Glasgow, Paris-Dauphine and St Andrews, and from conference participants at the Econometric Society World Congress (London, August 2005) and the Annual Meeting of the European Economic Association (Amsterdam, August 2005). All remaining errors are mine.

\section{Appendix: Heterogenous risk aversion}

This Appendix generalises our results to the case where risk aversion, in addition to entry costs, may differ across investors. More specifically, assume that investor $i$ is a CARA maximiser with risk-aversion coefficient $\gamma(i)$, where $\gamma(i) \in\left[\gamma_{\min }, \gamma_{\max }\right]$ and $0<\gamma_{\min }<\gamma_{\max }<\infty$. Investor $i$ now chooses the asset portfolio that approximately maximises

$$
E_{t}\left(-\exp \left(-\gamma(i) C_{t+1}^{i}\right)\right) \simeq \hat{a}^{i}+\hat{b}^{i}\left(E_{t}\left(C_{t+1}^{i}\right)-\frac{\gamma(i)}{2} \operatorname{Var}_{t}\left(C_{t+1}^{i}\right)\right)
$$

where $\hat{a}^{i} \equiv-(1+\gamma(i)) \exp \left(-\gamma(i) \bar{C}^{i}\right)$ and $\hat{b}^{i} \equiv \gamma(i) \exp \left(-\gamma(i) \bar{C}^{i}\right)$. The basic difference from the homogenous-preferences specification of Sec.3 is that optimal portfolios may now differ across investors. However, keeping the CARA assumption ensures that investors' demand for risky assets does not depend on investor wealth (although the latter may affect their utility through $\hat{a}^{i}$ and $\hat{b}^{i}$ ). Maximising (20) subject to (1) yields the following notional asset demand for investor $i$ :

$$
\hat{X}_{t}^{i}=\frac{E_{t}\left(p_{t+1}\right)+d-R p_{t}}{\gamma(i) \sigma_{t}^{2}} .
$$

We now rank investors in non-decreasing order of $\gamma(i) \epsilon(i)$. We further assume that both $\gamma(i)$ and $\epsilon(i)$ are continuous functions, and that $\epsilon(i)=0$ for $i \leq \tilde{\imath}$ and $\epsilon(i)>0$ for $i>\tilde{\imath}$, where $\tilde{\imath} \in(0,1)$, and that the function $h(i) \equiv \gamma(i) \epsilon(i)$ is increasing (and thus invertible) over $(\tilde{\imath}, 1]$. Comparing the utility from participation with that from staying out of the market as before, we find that investor $i$ participates if, and only if,

$$
\frac{\left(E_{t}\left(p_{t+1}\right)+d-R p_{t}\right)^{2}}{2 R \sigma_{t}^{2}} \geq \gamma(i) \epsilon(i) .
$$


The market-clearing condition now becomes:

$$
\int_{0}^{i_{t}} \hat{X}_{t}^{i} d i=\left(\frac{E_{t}\left(p_{t+1}\right)+d-R p_{t}}{\sigma_{t}^{2}}\right) \int_{0}^{i_{t}} \gamma(i)^{-1} d i=1,
$$

where $\int_{0}^{i_{t}} \gamma(i)^{-1} d i$ is continuous and strictly increasing in $i_{t}$. Solving (22) for the expected net payoff, $E_{t}\left(p_{t+1}\right)+d-R p_{t}$, and substituting the resulting expression into (5), we find that the sorting rule (analogue of Eq. (10)) is now:

$$
\begin{aligned}
\frac{\sigma_{t}^{2}}{2 R \gamma(i)\left(\int_{0}^{i_{t}} \gamma(i)^{-1} d i\right)^{2}} & \geq \epsilon(i) \text { for } i \leq i_{t} \\
& <\epsilon(i) \text { for } i>i_{t}
\end{aligned}
$$

In short, the latter inequality states that investor $i$ participates if, and only if, the expected utility from trading the asset outweighs the trading cost, $\epsilon(i)$. Greater risk aversion lowers the utility from trading the asset (given $\sigma_{t}^{2}$ and $\int_{0}^{i_{t}} \gamma(i)^{-1} d i$ ), thereby making entry relatively less valuable for investor $i$. Given the continuity assumptions maintained throughout, inequality (23) must hold as an equality for the marginal investor, which implies that the conditional variance of asset prices is related to participation as follows:

$$
\sigma_{t}^{2}=2 R \epsilon\left(i_{t}\right) \gamma\left(i_{t}\right)\left(\int_{0}^{i_{t}} \gamma(i)^{-1} d i\right)^{2}
$$

Solving (22) for $p_{t}$, using (24), and then iterating forwards the resulting expression while focusing on non-explosive price solutions, we find that the level of asset prices is now:

$$
p_{t}=\frac{d}{R-1}-2 E_{t}\left(\sum_{j=0}^{\infty} R^{-j} \epsilon\left(i_{t}\right) \gamma\left(i_{t}\right) \int_{0}^{i_{t}} \gamma(i)^{-1} d i\right) .
$$

We can construct stochastic equilibria for the heterogenous risk-aversion economy in an analogous manner to Sec. 3.2 for the homogenous risk-aversion case. More specifically, define the continuous function $J_{t}=g\left(i_{t}\right) \equiv 2 \epsilon\left(i_{t}\right) \gamma\left(i_{t}\right) \int_{0}^{i_{t}} \gamma(i)^{-1} d i$, where by construction $g\left(i_{t}\right)=0$ for $i_{t} \leq \tilde{\imath}$ and $g\left(i_{t}\right)>0, g^{\prime}\left(i_{t}\right)>0$ for $i_{t}>\tilde{\imath}$ (so that $J_{t}$ is uniquely defined by $i_{t}$ for $i_{t}>\tilde{\imath}$ ). Assume that $i_{t}>\tilde{\imath} \forall t$, and that $J_{t}$ evolves according to $J_{t}=\bar{J}+G\left(J_{t-1}\right) \eta_{t}$, where $\bar{J}$ is the mean of $\left\{J_{t}\right\}_{t=0}^{\infty},\left\{\eta_{t}\right\}_{t=0}^{\infty}$ is as in Sec. 3.2, and $G\left(J_{t-1}\right)$ is a sensitivity function. Substituting the $J_{t} \mathrm{~s}$ into the price process, we may rewrite the latter as:

$$
p_{t}=(d-\bar{J}) /(R-1)-J_{t},
$$


implying that the conditional variance of prices is $\sigma_{t}^{2}=G\left(J_{t-1}\right)^{2}$. The sensitivity function $G\left(J_{t-1}\right)$ must be such that $(24)$ holds in every period. From the definitions of $g($.$) and h($. (both of which being continuously increasing, and thus invertible, over $(\tilde{\imath}, 1]$ ), the variance in (24) can be written as $\sigma_{t}^{2}=R J_{t}^{2} / 2 h\left(g^{-1}\left(J_{t}\right)\right)$, giving the sensitivity function:

$$
G\left(J_{t}\right)=\sqrt{\frac{R J_{t}^{2}}{2 h\left(g^{-1}\left(J_{t}\right)\right)}} .
$$

We must first establish that $G(J)$ is strictly increasing, so that $G(J)$ reaches its upper bound when $J$ does. $G^{\prime}(J)>0$ is equivalent to $2 h\left(g^{-1}(J)\right)>J h^{\prime}\left(g^{-1}(J)\right) \times \partial g^{-1}(J) / \partial J$. Since $J=g(i)$, and thus $\partial g^{-1}(J) / \partial J=1 / g^{\prime}(i)$, the previous inequality can be rearranged to yield $2 h(i)>g(i) h^{\prime}(i) / g^{\prime}(i)$. Now, using the fact that $g(i) / 2 h(i)=\int_{0}^{i} \gamma(x)^{-1} d x$, we find that $G^{\prime}(J)>0$ is increasing if (and only if) $g^{\prime}(i)>h^{\prime}(i) \int_{0}^{i} \gamma(x)^{-1} d x$, which is always true since $g^{\prime}(i)=2 h^{\prime}(i) \int_{0}^{i} \gamma(x)^{-1} d x+2 h(i) \int_{0}^{i} \gamma(x)^{-1} d x$.

The upper bound is the solution $J^{*}$ to $J=\bar{J}+G(J) \hat{\eta}$ or, using (26) and rearranging, to

$$
\mu(J) \equiv 1-\bar{J} / J=\hat{\eta} \sqrt{R / 2 h\left(g^{-1}(J)\right)} \equiv \tau(J),
$$

where we let $J$ vary over $[\bar{J}, g(1)] . \mu($.$) is strictly increasing and \mu(\bar{J})=0$, while $\tau($.$) is$ strictly decreasing and $\tau(\bar{J})>0$. Thus, $J^{*}$ exists and is unique if $\mu(g(1))>\tau(g(1))$, that is, if $g(1)-\bar{J}>\hat{\eta} \sqrt{R g(1)^{2} / 2 h(1)}$. From the definitions of $g($.$) and h($.$) , this gives:$

$$
2 \epsilon(1) \gamma(1) \int_{0}^{1} \gamma(i)^{-1} d i-\bar{J}>\hat{\eta} \sqrt{2 R \epsilon(1) \gamma(1)\left(\int_{0}^{1} \gamma(i)^{-1} d i\right)^{2}} .
$$

$J_{t}$ is bounded below by $J_{*}=\bar{J}-G\left(J^{*}\right) \hat{\eta}$. Since $J^{*}=\bar{J}+G\left(J^{*}\right) \hat{\eta}$, the condition that $J_{*}>0$ is equivalent to $2 \bar{J}>J^{*}$. Now, since $J^{*}<g(1)$, choosing $\bar{J} \geq g(1) / 2$ ensures that $J_{t}$ never goes below 0 . Then, setting $\bar{J}=g(1) / 2$ and $\hat{\eta}=1$ in (27), we find that a sufficient condition for $J_{t}$ to stay within the required interval is $\gamma(1) \epsilon(1)>2 R$. 


\section{References}

Aiyagari, S.R., 1988. Economic fluctuations without shocks to fundamentals; Or, does the stock market dance to its own music? Federal Reserve Bank Minneapolis Quarterly Review 12, 8-24.

Allen, A., Gale, D., 1994. Limited participation and volatility of asset prices. American Economic Review 84, 933-955.

Azariadis, C., Kaas, L., 2007. Asset price fluctuations without aggregate shocks. Journal of Economic Theory 136, 126-143.

Azariadis, C., Chakraborty, S., 1998. Asset price volatility in a nonconvex general equilibrium model. Economic Theory 12, 649-665.

Benhabib, J., Farmer, R.E.A., 1999. Indeterminacy and sunspots in macroeconomics, in: Taylor, J., Woodford, M. (Eds.), Handbook of Macroeconomics, North Holland, pp. 387-448.

Benhabib, J., Farmer, R.E.A., 1994. Indeterminacy and increasing returns. Journal of Economic Theory 63, 19-41.

Campbell, J., Shiller, R.J., 1989. The dividend-price ratio and expectations of future dividends and discount factors. Review of Financial Studies 1, 195-227.

Challe, E., 2004. Sunspots and predictable asset returns. Journal of Economic Theory 115, 182-190.

Constantinides, G.M., Donaldson, J.B., Mehra, R., 2002. Junior can't borrow: A new perspective on the equity premium puzzle. Quarterly Journal of Economics 117, 269-296.

Engle, R., 2004. Risk and volatility: Econometric models and financial practice. American Economic Review 94, 405-420.

Guiso, L., Halliasos, M., Jappelli, T., 2003. Household stockholding in Europe: Where do we stand, and where do we go? Economic Policy 18, 123-170.

Hanoch, G., Levy, H., 1969. The efficiency analysis of choices involving risk. Review of Economic Studies 36, 335-346.

Hellwig, M.F., 1982. Rational expectations equilibrium with conditioning on past prices: A mean-variance example. Journal of Economic Theory 26, 279-312.

Jones, C., Kaul, G., Lipson, M.L., 1994. Transactions, volume, and volatility. Review of Financial Studies 7, 631-651. 
Karpoff, M., 1987. The relation between price changes and trading volume: A survey. Journal of Financial and Quantitative Analysis 22, 109-126.

Levy, H., Markovitz, H.M., 1979. Approximating expected utility by a function of mean and variance. American Economic Review 69, 307-317.

Mankiw, G., Zeldes, S., 1991. The consumption of stockholders and nonstockholders. Journal of Financial Economics 29, 97-112.

Orosel, G.O., 1998. Participation costs, trend chasing, and volatility of stock prices. Review of Financial Studies 11, 521-557.

Pagano, M., 1989a. Endogenous market thinness and stock price volatility. Review of Economic Studies 56, 269-287.

Pagano, M., 1989b. Trading volume and asset liquidity. Quarterly Journal of Economics $104,255-274$.

Poterba, J.M., Summers, L.H., 1986. The persistence of volatility and stock market fluctuations. American Economic Review 76, 1142-1151.

Poterba, J.M., Summers, L.H., 1988. Mean reversion in stock prices: Evidence and implications. Journal of Financial Economics 22, 26-59.

Shiller, R.J., 1981. Do stock prices move too much to be justified by subsequent changes in dividends? American Economic Review 71, 421-436.

Spiegel, M., 1998. Stock-price volatility in a multiple security overlapping generations model. Review of Financial Studies 11, 419-447.

Woodford, M., 1986. Stationary sunspot equilibria: The case of small fluctuations around a deterministic steady state. Unpublished manuscript. 


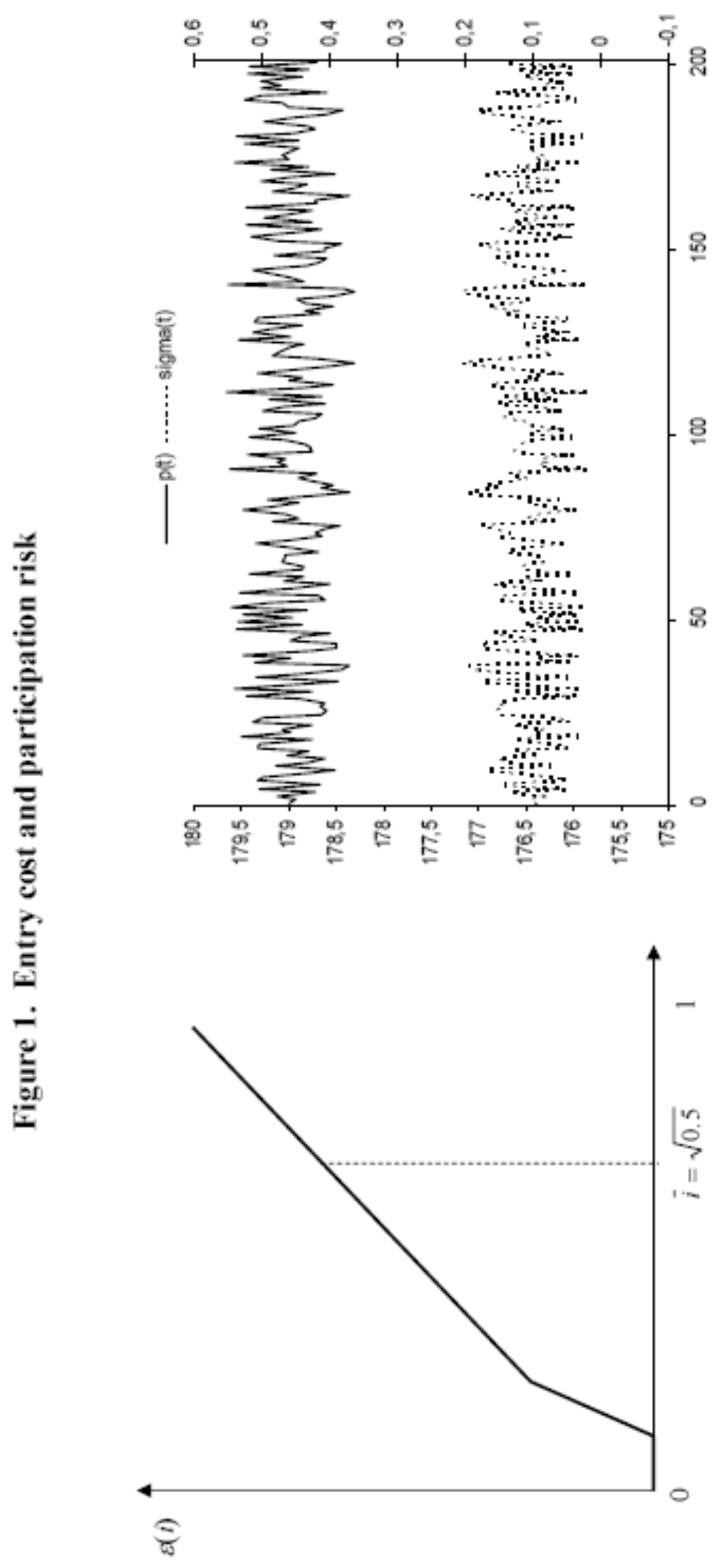

